\title{
Correspondence
}

\section{Fetal blood gas analysis at delivery}

Sir,

In a recent article D'Souza et al. ${ }^{1}$ note that it is simpler and more convenient to obtain umbilical venous than arterial blood to assess the acid base status of a newborn infant at delivery. They add, however, that sampling arterial blood would be preferable to venous blood as the former reflects more closely the metabolic condition of the fetus. This clinical dilemma can be resolved very easily by sampling blood from the fetal arteries that cross the chorionic plate of the placenta. As in the retina of the eye the arteries can be accurately identified as they cross over the veins.

For a number of years we have obtained fetal arterial blood in this manner to determine the degree of metabolic acidosis in infants born depressed or after an obstetric diagnosis of fetal distress. The method is easy, especially if the fetal blood is not permitted to drain before delivery of the placenta.

The measurement of base excess or buffer base is preferred to $\mathrm{pH}$ as the latter is rapidly altered by changes in maternal $\mathrm{PCO}_{2}$. For exemple, the presence of hypocarbia provides the explanation of the finding of an alkalosis in many of the infants studied by D'Souza et al. Lastly it remains debatable whether a maternal metabolic acidosis influences the acid base status of the infant at birth. $^{2}$

\section{L Woods Department of Paediatrics and Child Health, University of Cape Town Medical School, Observatory, Cape, 7925, South Africa}

\section{Drs D'Souza and Richards comment:}

Obtaining a sample of blood from fetal arteries crossing the chorionic surface of the placenta may be feasible but we are concerned that this blood is less likely to reflect the $\mathrm{pH}, \mathrm{PO}_{2}$, and $\mathrm{PCO}_{2}$ in the infant at birth, since there is a delay in the delivery of the placenta. Several minutes generally lapse between birth of the infant and delivery of the placenta during which time fetal blood will not be circulating in the placenta after the cord is clamped. This raises some uncertainty about the relevance of $\mathrm{pH}, \mathrm{Po}_{2}$, and $\mathrm{PCO}_{2}$ measurements in fetal blood from the placenta.

In our study base excess was calculated from blood $\mathrm{pH}$, $\mathrm{PCO}_{2}$, and haemoglobin. There is no way that base excess can be measured, it can only be calculated from $\mathrm{pH}, \mathrm{PCO}_{2}$, and haemoglobin. One can either use the Siggard-Andersen alignment nomogram ${ }^{3}$ or one of the several equations, for example Siggard-Andersen ${ }^{4}$; both these are incorporated in the Radiometer slide rule ${ }^{5}$ and microcomputer chips incorporated into many modern blood gas machines (for example, Corning) ${ }^{6}$ but all demand a knowledge of the haemoglobin value. To follow Dr Woods's path necessitates measuring the 3 parameters, especially haemoglobin, and measuring them quickly. Additional information obtained by calculating base excess is useful in that buffer base is altered depending on whether there is a metabolic acidosis, as in accumulation of lactate in the fetus, or a non-metabolic acidosis resulting from carbon dioxide retention.

Blood pH and gas tensions were not measured in our mothers during labour but we have referred to the results of other studies in which such measurements have been carried out. In newborn infants we found that blood $\mathrm{pH}$, $\mathrm{Po}_{2}$, and $\mathrm{PCO}_{2}$ measurements can be conveniently carried out when Astrup apparatus is available on the delivery unit. Lactate concentrations are another indicator of asphyxia at birth, but lactate measurements are less convenient since it is necessary to send blood specimens to the laboratory. Delay in obtaining results would reduce the usefulness of such measurements in the diagnosis and management of asphyxia at birth.

\section{References}

1 D'Souza SW, Black P, Cadman J, Richards B. Umbilical venous blood pH: a useful aid in the diagnosis of asphyxia at birth. Arch Dis Child 1983; 58: 15-9.

2 Dellenbach P, Haberey P. Lactate as indicator for fetal and neonatal asphyxia (letter). Lancet 1982; i: 907.

3 Siggaard-Anderson $O$. Blood acid-base alignment nomogram. Scand J Clin Lab Invest 1963; 15: 211-7.

4 Siggaard-Anderson O. The acid-base status of the blood. 4th ed. Copenhagen: Munksgaard, 1974.

5 Severinghaus JW. Blood gas calculator. J Appl Physiol $1966 ; 21$ : 1108-16.

6 The Instruction Manual 175 Automatic Blood Gas Analyser. Corning Medical, 1976.

\section{Ventricular enlargement after periventricular haemorrhage}

Sir,

Palmer et al..$^{1}$ have shown that in 25 preterm infants surviving periventricular haemorrhage, cerebral ventricular enlargement was significantly associated with a poorer outcome during the first year of life and that this effect was irrespective of the size of the initial haemorrhage. I have followed up 67 very low birthweight infants born in 1980 and 1981 who survived periventricular haemorrhage. Twenty eight showed ventricular enlargement, with ventricular width exceeding $5 \mathrm{~mm}$ at the level of the lateral sulcus on a coronal ultrasound scan via the anterior fontanelle. Follow up examination using a neurological examination, Denver developmental screening test, and Stycar tests of hearing and vision showed 26 infants to have a neurological deficit or developmental delay, or both at 18 months to 3 years. Eight of these infants have a ventriculo-peritoneal shunt inserted for progressive hydrocephalus. 